\title{
Hybrid procedure in living donor liver transplantation
}

Akihiko Soyama, Mitsuhisa Takatsuki, Masaaki Hidaka, Tomohiko Adachi,

Amane Kitasato, Ayaka Kinoshita, Koji Natsuda, Zhassulan Baimakhanov,

Tamotsu Kuroki, Susumu Eguchi

Department of Surgery, Nagasaki University Graduate School of Biomedical Sciences

Keywords: living donor, laparoscopic, less invasive surgery, transplantation

Address correspondence to: Susumu Eguchi, MD.

Department of Surgery, Nagasaki University Graduate School of Biomedical Sciences, 1-7-1

Sakamoto, Nagasaki 852-8501, Japan

TEL: 81-95-819-7316

FAX: 81-95-819-7319

E-mail: sueguchi@nagasaki-u.ac.jp 


\section{Abstract \\ Background}

We have previously reported a hybrid procedure that uses a combination of laparoscopic mobilization of the liver and subsequent hepatectomy under direct vision in living donor liver transplantation (LDLT). We present the details of this hybrid procedure and the outcomes of the procedure.

Methods

Between January 1997 and August 2014, 204 LDLTs were performed at Nagasaki University Hospital. Among them, 67 recent donors underwent hybrid donor hepatectomy. Forty-one donors underwent left hemihepatectomy, 25 underwent right hemihepatectomy, and 1 underwent posterior sectionectomy. First, an 8-cm subxiphoid midline incision was made; laparoscopic mobilization of the liver was then achieved with a hand-assist through the midline incision under the pneumoperitoneum. Thereafter, the incision was extended up to $12 \mathrm{~cm}$ for the right lobe and posterior sector graft and $10 \mathrm{~cm}$ left lobe graft procurement. Under direct vision, parenchymal transection was performed by means of the liver-hanging maneuver. The hybrid procedure for 
LDLT recipients was indicated only for selected cases with atrophic liver cirrhosis without a history of upper abdominal surgery, significant retroperitoneal collateral vessels, or hypertrophic change of the liver $(n=29)$. For total hepatectomy and splenectomy, the midline incision was sufficiently extended.

Results

All of the hybrid donor hepatectomies were completed without an extra subcostal incision.

No significant differences were observed in the blood loss or length of the operation compared with conventional open procedures. All of the donors have returned to their preoperative activity level, with fewer wound-related complaints compared with those treated with the use of the conventional open procedure. In recipients treated with the hybrid procedure, no clinically relevant drawbacks were observed compared with the recipients treated with a regular Mercedes-Benz-type incision

Conclusions

Our hybrid procedure was safely conducted with the same quality as the conventional open procedure in both LDLT donors and recipients. 


\section{Introduction}

Applications of less invasive techniques, including laparoscopic procedures, have been reported in the field of living donor liver transplantation (LDLT)(1-3). We have reported a hybrid procedure employing a combination of hand-assisted laparoscopic mobilization of the liver and subsequent hilar dissection and parenchymal resection under direct vision in living donor hepatectomy $(1,4)$. In terms of the appearance, sensation and daily activities, our hybrid procedure was found to have a better donor self-assessment compared with those treated with a conventional incision, like a right subcostal incision or Mercedes-Benz incision (5).

We also introduced the basic concept of the hybrid procedure into recipient surgery in selected cases (6). We herein present the current practice of the hybrid procedure and the outcomes of the procedures in LDLT. 


\section{Methods}

The hybrid procedure for LDLT donors

Between January 1997 and August 2014, 204 patients underwent LDLT at Nagasaki University

Hospital. Among them, 67 recent donors underwent hybrid donor hepatectomy. Forty-one donors

underwent left hemihepatectomy; 25 underwent right hemihepatectomy and one underwent

posterior sectionectomy. We compared the surgical outcomes, including the blood loss, length of the

operation and postoperative complications classified according to the Clavien-Dindo classification

(7) between the donors who underwent hybrid donor hepatectomy and conventional open

procedures.

The hybrid procedure is a combination of a laparoscopic procedure and an open procedure.

The laparoscopic procedure includes hand-assisted mobilization of the liver and the subsequent

open procedure with an upper midline incision comprises vessel management, parenchymal

resection and graft removal. During the procedure, an 8-cm subxiphoid midline incision is first

created for inspection of the liver and subsequent hand assistance during mobilization of the liver.

After sufficient mobilization of the liver, the aforementioned subxiphoid incision was basically 
extended to $12 \mathrm{~cm}$ for the right hemihepatectomy and $10 \mathrm{~cm}$ for a left hemihepatectomy. However, since minimizing the incision is not the main objective of this procedure, if any difficulty was expected for surgery with a 10-12 cm incision, the incision was extended without any hesitation. Encircling the hepatic veins and hilar dissection were performed under direct vision. Parenchymal resection was performed with the liver-hanging maneuver. Bile duct division was performed after visualizing the planned transection point by encircling the bile duct using a radiopaque marker filament under real-time C-arm cholangiography (8). Further details of the procedure were described elsewhere $(1,4)$.

Although we used a vascular clamp when transecting the hepatic veins in the early cases, as a modification of the procedure, we are currently using a triple-lined vascular stapler for transection of the hepatic vein to prevent accidental slipping off of the vascular clamp. Using the vascular stapler had made graft removal even safer, while preserving a sufficient length of hepatic vein cuff for anastomosis.

The hybrid procedure for LDLT recipients 
The hybrid procedure for LDLT recipients was indicated only for selected cases without a

previous history of upper abdominal surgery, significant retroperitoneal collateral vessels or hypertrophic changes of the liver. Furthermore, patients with a deep location of the venous anastomosis from the body surface were considered to be difficult treated using the hybrid procedure. The laparoscopic procedure includes hand-assisted mobilization of the liver, and also the spleen when splenectomy is indicated. After the bilateral mobilization of the liver and spleen, the midline incision is extended to just above the navel for subsequent procedures, including total hepatectomy and implantation. In total, 29 patients underwent this procedure during living donor liver transplantation. The surgical outcomes were evaluated and compared with those in patients who underwent conventional procedures.

\section{Statistics}

Mann-Whitney U-test or chi-suqare test was applied to compare the groups where appropriate. P-value less than 0.05 was considered statistically significant. 


\section{Results}

The hybrid procedure in LDLT donors

All of the hybrid donor hepatectomies were completed without an extra subcostal incision.

When comparing the donors with hybrid hepatectomies and open procedures, besides types of

hepatectomies, no significant differences were recognized in their characteristics including age,

gender, BMI, the type of procedure, renal function, and gender mismatch with the recipient (Table

1). No donor underwent left lateral sectionectomy by the hybrid procedure. The renal function

was evaluated based on the estimated GFR which was calculated using a formula for the Japanese

population recommended by the Japanese Society of Nephrology (9). When the findings of the

hybrid procedure were compared with those of the open procedure for living donor left

hemihepatectomy (hybrid group, $n=41$, open group, $n=39$ ) and right hemihepatectomy ( $n=25$ per

group), no significant differences were seen in the duration of the operation or in the blood loss

(Table 2). The median duration of the operation for the hybrid right hemihepatectomy was 411 min

(range, 324-581), and that of the left hemihepatectomy was 401 min (range 286-671), with some

adjustment period between the donors and the recipients. The median blood loss in the hybrid right 
hemihepatectomy was $600 \mathrm{~g}$ (range, 130-1,900), and that for the left hemihepatectomy was $475 \mathrm{~g}$

(range 50-3,350), including blood from the cuff of the hepatic veins from the graft. No donors treated with the hybrid procedure required an allogeneic transfusion.

All of the donors have returned to their preoperative activity level with fewer wound-related complaints, such as numbness in the abdominal wall, compared to those treated using the conventional open procedure during the long-term follow-up (Fig. 1). With respect to morbidity, no significant differences were recognized between the hybrid group and open group (Table 3).

The hybrid procedure in LDLT recipients

No significant differences were seen in the blood loss, duration of vascular anastomosis and whole procedures between the groups treated with the hybrid procedure and those treated with the conventional open procedure (Table 4). Simultaneous splenectomy was more frequently performed in recipients with the hybrid procedure. Although the median follow-up periods were different, no significant difference has so far been seen in the survival between patients after the conventional open and hybrid procedures (data not shown). 


\section{Discussion}

According to our experiences with both LDLT donors and recipients who underwent the hybrid procedure, no clinically important drawbacks were observed. In the donor procedure, after sufficient mobilization of the liver, sufficiently wide surgical fields are available through the upper midline incision without the need for abdominal muscle disruption. For the mobilization of the liver through the upper midline incision, there have been arguments about the necessity of a laparoscopic procedure. Indeed, several authors have reported living donor right or left hemihepatectomy with an upper midline incision $(10,11)$. The reason why we use a laparoscopic procedure is that laparoscopic mobilization of the liver makes the donor hepatectomy possible through a midline incision, regardless of the constitution of the donor, such as a narrow subcostal angles or a deep abdominal cavity. Since the median duration of hand-assisted laparoscopic mobilization of the liver was about 30 minutes in the hybrid procedure (4), the procedure did not prolong the total operation.

Because LDLT is basically performed in an elective manner, when taking into account the duration of the laparoscopic procedure, it seems reasonable to prepare for a hand-assisted laparoscopic procedure from the beginning of surgery without exception, not only in cases where mobilization 
through an upper midline incision is expected to be difficult.

The total length of the operation was not significantly different between the hybrid group and conventional open group. No negative impressions about the laparoscopic procedure in terms of the length of the operation exist for our procedure.

Under direct vision, a safe and accurate procedure with the same quality as the conventional open procedure using the liver hanging maneuver and real-time c-arm cholangiography can be performed. In terms of invasiveness, it is important to recognize that this method is associated with fewer complaints about scarring. We have investigated the postoperative self-assessments concerning surgical scars in 87 living donors treated with three types of incisions for donor hepatectomy (5). The investigation revealed that numbness of the abdominal wall was reported more frequently by the donor treated with a Mercedes-Benz incision or right subcostal incision up to xiphoid incisions compared with donors treated with an upper midline incision (5). Since the publication of that report, the total number of donors who underwent hybrid donor hepatectomy with a midline incision has increased up to 68 from 15. Among them, 36 donors underwent self-assessment of the postoperative scars. The results showed significantly less numbness in donors 
treated with a midline incision than donors treated with a Mercedes-Benz incision or right subcostal incision, confirming the finding of our previous report (5). Recently, Suh et al. reported the patient satisfaction in living liver donors according to differences in the incisions used for donor hepatectomy (12). In that study, the satisfaction levels of the patients treated with an upper midline incision or transverse incision using laparoscopy were higher and had improved cosmetic outcomes compared to cases treated with a conventional incision (12).

Since LDLT is usually performed in an elective manner, the hybrid procedure could be planned and prepared for. Laparoscopic splenectomy with or without hand-assist has become a standard procedure. This concept can be introduced into splenectomy during LDLT, as we have done in the hybrid procedure. Judging from the outcomes, our indication seems reasonable. Because no muscle disruption occurs during this procedure, improved postoperative rehabilitation is expected. Further investigation about the post-transplant recovery following the hybrid procedure is needed.

In conclusion, our hybrid living donor hepatectomy is considered to be a reasonable procedure, including the merits of both laparoscopic and open procedures. We will continue to 
improve this procedure after carefully evaluating the early postoperative and long-term outcomes.

\section{References}

1. Soyama A, Takatsuki M, Hidaka M, et al. Standardized less invasive living donor hemihepatectomy using the hybrid method through a short upper midline incision. Transplant Proc 2012;44:353-5.

2. Troisi RI, Wojcicki M, Tomassini F, et al. Pure laparoscopic full-left living donor hepatectomy for calculated small-for-size LDLT in adults: proof of concept. Am J Transplant 2013;13:2472-8.

3. Koffron AJ, Kung R, Baker T, et al. Laparoscopic-assisted right lobe donor hepatectomy. Am J Transplant 2006;6:2522-5.

4. Soyama A, Takatsuki M, Adachi T, et al. A hybrid method of laparoscopic-assisted open liver resection through a short upper midline laparotomy can be applied for all types of hepatectomies. Surg Endosc 2014;28:203-11.

5. Imamura H, Soyama A, Takatsuki M, et al. Self-assessment of postoperative scars in living liver donors. Clin Transplant 2013;27:E605-10.

6. Eguchi S, Takatsuki M, Soyama A, et al. Elective living donor liver transplantation by hybrid hand-assisted laparoscopic surgery and short upper midline laparotomy. Surgery 2011;150:1002-5.

7. Dindo D, Demartines N, Clavien PA. Classification of surgical complications: a new proposal with evaluation in a cohort of 6336 patients and results of a survey. Ann Surg 2004;240:205-13.

8. Takatsuki M, Eguchi S, Yamanouchi K, et al. Technical refinements of bile duct division in living donor liver surgery. J Hepatobiliary Pancreat Sci 2011;18:170-5.

9. Matsuo S, Imai E, Horio M, et al. Revised equations for estimated GFR from serum creatinine in Japan. Am J Kidney Dis 2009;53:982-92.

10. Nagai S, Brown L, Yoshida A, et al. Mini-incision right hepatic lobectomy with or 
without laparoscopic assistance for living donor hepatectomy. Liver Transpl 2012;18:1188-97.

11. Lee KW, Kim SH, Han SS, et al. Use of an upper midline incision for living donor partial hepatectomy: a series of 143 consecutive cases. Liver Transpl 2011;17:969-75.

12. Suh SW, Lee KW, Lee JM, et al. Clinical outcomes of and patient satisfaction with different incision methods for donor hepatectomy in living donor liver transplantation. Liver Transpl 2015;21:72-8. 


\section{Fig. 1}

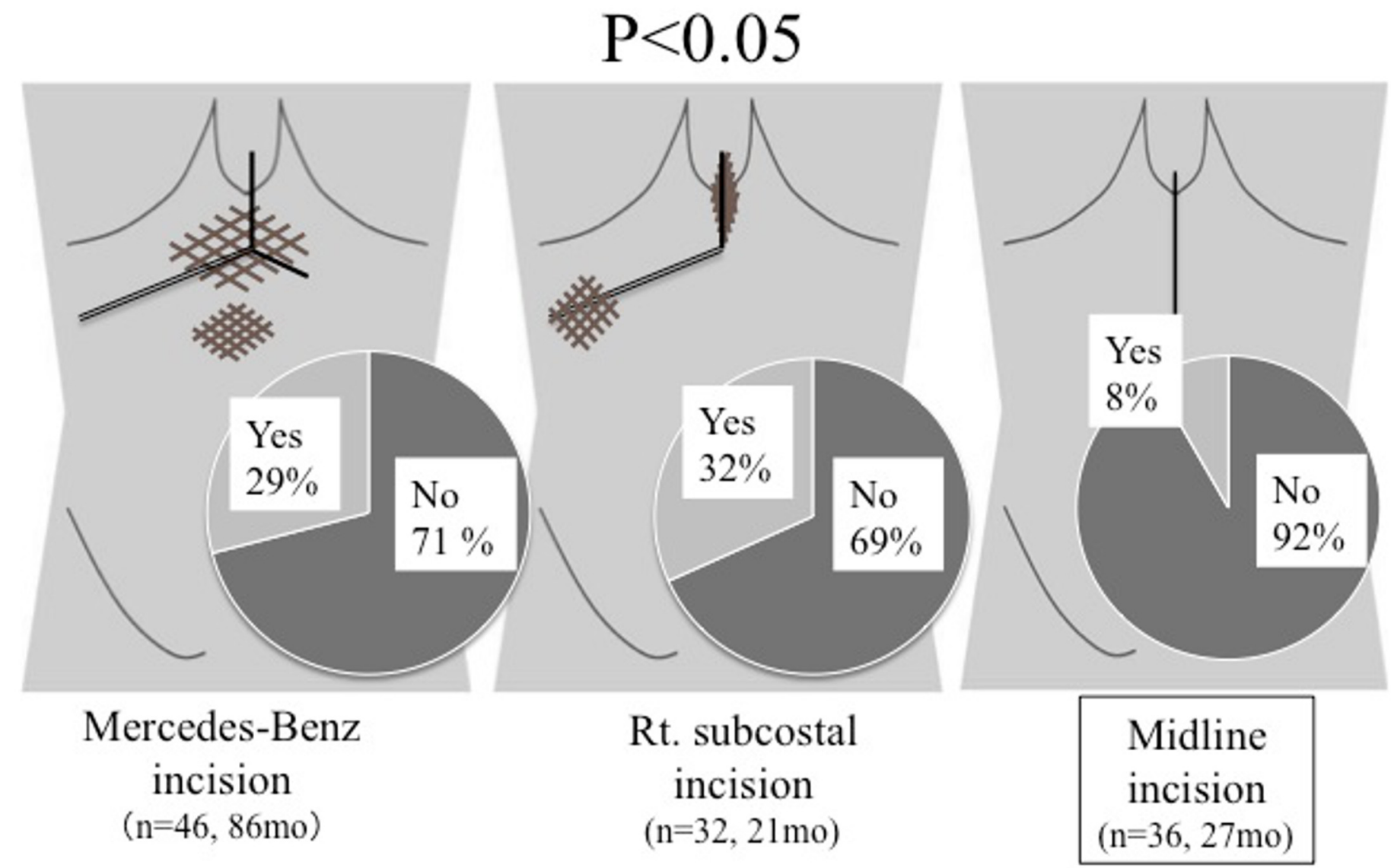

Results of an evaluation of abdominal wall numbness according to type of postoperative scar with the use of a self-assessment questionnaire. 
Table 1. A comparison of the demographics of the hybrid and conventional open procedures in living liver donors

\begin{tabular}{lccc}
\hline & Hybrid $(\mathrm{n}=67)$ & Open $(\mathrm{n}=137)$ & $P$-value \\
\hline Age (median, range) & $41(21-65)$ & $39(19-67)$ & N.S. \\
Gender $($ Male:Female) & $33: 34$ & $57: 80$ & N.S. \\
BMI $\left(\mathrm{kg} / \mathrm{m}^{2}\right)$ & $21.6(16.9-29.0)$ & $22.1(16.4-34.7)$ & N.S. \\
Type of procedure & & & \\
$\quad$ Right hemihepatectomy & 25 & 59 & \\
$\quad$ Left hemihepatectomy & 41 & 60 & $<0.05$ \\
$\quad$ Right posterior sectionectomy & 1 & 6 & \\
Left lateral sectionectomy & 0 & & \\
& & & \\
Gentimated GFR (ml $/$ min $\left./ 1.73 \mathrm{~m}^{2}\right)$ & $85.4(59.2-139.3)$ & $87.6(54.1-143.2)$ & N.S. \\
Ger mismatches with recipients & $39 / 67(58 \%)$ & $68 / 137(50 \%)$ & N.S. \\
\hline
\end{tabular}


Table 2. A comparison of the surgical outcomes of the hybrid and open procedures for living-donor hemihepatectomy

\begin{tabular}{|c|c|c|c|}
\hline & Hybrid & Open & $P$-value \\
\hline Right hemihepatectomy & $\mathrm{n}=25$ & $\mathrm{n}=25$ & \\
\hline $\begin{array}{l}\text { Duration of surgery } \\
\text { (min) }\end{array}$ & 411 (324-581) & $415(350-523)$ & N.S. \\
\hline Blood loss (g)* & $600(130-1900)$ & $687(140-1800)$ & N.S. \\
\hline Left hemihepatectomy & $\mathrm{n}=41$ & $\mathrm{n}=39$ & \\
\hline $\begin{array}{l}\text { Duration of surgery } \\
\text { (min) }\end{array}$ & $398(286-671)$ & $400(310-802)$ & N.S. \\
\hline Blood loss $(\mathrm{g})^{*}$ & $475(50-3350)$ & $610(170-3150)$ & N.S. \\
\hline
\end{tabular}


Table 3. The postoperative complications according to the Clavien-Dindo classification

\begin{tabular}{|c|c|c|c|c|}
\hline & & Total $(n=204)$ & Hybrid $(n=67)$ & Open $(n=137)$ \\
\hline \multirow[t]{7}{*}{ Grade I } & & Bile leakage & 2 & 6 \\
\hline & & Pleural effusion & & 2 \\
\hline & & Wound infection & & 2 \\
\hline & & Median nerve paralysis & & 1 \\
\hline & & Recurrent nerve paralysis & & 1 \\
\hline & & Drain site infection & & 1 \\
\hline & & Postoperative bleeding & 2 & \\
\hline Grade II & & $\begin{array}{l}\text { Postoperative bleeding } \\
\text { Acute pancreatitis }\end{array}$ & & $\begin{array}{l}1 \\
1 \\
-1\end{array}$ \\
\hline \multirow[t]{7}{*}{ Grade III } & IIIa & Bile leakage & & 4 \\
\hline & & Skin necrosis & & 1 \\
\hline & & Gastric stasis & & 3 \\
\hline & & Bleeding of duodenal ulcer & 1 & \\
\hline & IIIb & Gastric stasis & & 1 \\
\hline & & PV thrombus & 1 & \\
\hline & & Ileus & 1 & \\
\hline \multirow[t]{2}{*}{ Grade IV } & IVa & PV thrombus & & 1 \\
\hline & $\mathrm{IVb}$ & 0 & & \\
\hline Grade V & & 0 & & \\
\hline Total & & $32(16 \%)$ & $7(10 \%)$ & $25(19 \%)$ \\
\hline
\end{tabular}


Table 4. A comparison of the patient demographics and surgical outcomes of the hybrid and conventional open procedures in LDLT recipients

\begin{tabular}{llll}
\hline & Hybrid $(\mathrm{n}=29)$ & $\begin{array}{l}\text { Conventional: } \\
\text { Mercedes-Benz } \\
\text { incision }(\mathrm{n}=29)\end{array}$ & $P$-value \\
\hline BMI $\left(\mathrm{kg} / \mathrm{m}^{2}\right)$ & $24.9(20.1-35.9)$ & $24.4(17.7-35.9)$ & N.S. \\
MELD score & $15(7-43)$ & $16(7-41)$ & N.S. \\
Graft type & Left $22:$ Right 7 & Left $20:$ Right 9 & N.S. \\
GW/SLV (\%) & $34.2(22.5-54.3)$ & $37.6(28.2-58.0)$ & N.S. \\
$\begin{array}{l}\text { Blood loss (g) } \\
\text { Duration of surgery (min) }\end{array}$ & $7300(1300-18400)$ & $4810(520-26500)$ & N.S. \\
$\begin{array}{l}\text { Duration of } \\
\text { vascular anastomosis (min) }\end{array}$ & $40(31-67)$ & $805(561-1159)$ & N.S. \\
$\begin{array}{l}\text { Simultaneous splenectomy } \\
\text { (5) }\end{array}$ & $23 / 29(79 \%)$ & $39(26-67)$ & N.S. \\
\hline
\end{tabular}

